Materials and Methods: The medical charts of 16 patients who underwent DCS from October 2000-April 2004 were reviewed retrospectively. All of the patients were classified into one of two groups: (1) Survived (Group A: 11 cases); and (2) Expired (Group B: 5 cases). The number of patients who underwent DCS, and met the following criteria were evaluated: (1) systolic blood pressure $<90 \mathrm{mmHg}$; body temperature $<35^{\circ} \mathrm{C}$; and (3) $\mathrm{pH}<7.3$.

Results: The aims of the DCS were hemorrhage control for massive hepatic injury in 12 cases (Group A: 7, Group B: 5) or severe pancreatic injury in four cases (all in Group A). The response pattern of the circulation from initial fluid resuscitation revealed transient responders (six cases) or non-responders (10 cases). The severity of injury was greater in B group (ISS of 39.4 and $p=0.47$ ) comparing with Group A (ISS of 31.3 and $p=0.729$ ). A matching number of these DCS criteria in Group A were 0 (3 cases), 1 ( 3 cases), 2 ( 5 cases) and 3 ( 1 case). On the other hand, all cases matched 3 (full scores) in Group B. The time from admission to fulfill these three criteria between both groups was 40.7 minutes.

Conclusion: Although further investigation is needed, the revised DCS decision criteria seems to be useful, and DCS should be performed before the abdominal trauma patient fulfills these three criteria.

Keywords: abdominal; acidosis; coagulopathy; criteria; damage control surgery (DCS); emergency department; fluid; hypotension; hypothermia; liver; pancreas; resuscitation; trauma

Prebosp Disast Med 2005;20(3):s146-s147

\section{Is Mechanism of Injury a Useful Predictor in} Prehospital Trauma Triage?

F. Archer, ${ }^{1}$ M. Boyle, ${ }^{1}$ E. Smith; ${ }^{1}$ P. Cameron; ${ }^{2}$ I. Patrick, ${ }^{3}$ T. Walker ${ }^{4}$

1. Monash University Centre for Ambulance and Paramedic Studies, Australia

2. Monash University, Department of Epidemiology and Preventive Medicine, Victoria, Australia

3. Metropolitan Ambulance Service (Melbourne), Victoria, Australia

4. Rural Ambulance Victoria, Australia

This project set out to answer a significant prehospital care question that remained unresolved following the 1999 Review of Trauma and Emergency Services in Victoria, "Is mechanism of injury a useful predictor in prehospital trauma triage?" The performance of triage in physiologically stable, trauma patients for whom only mechanistic criteria are present upon which to make judgments, has a high potential for over-triage; over-estimation of severity of injuries is unavoidable since there is a possibility that potentially serious injuries will be missed. Validation of the predictive value of application of mechanistic triage criteria has been limited in international literature, and hence, its value in predicting the severity of the injuries remains controversial.

However, prehospital trauma triage is a keystone of an effective, regional trauma system. This paper outlines the process and results of a study of the accuracy of the mechanistic triage criteria that was undertaken in a statewide emergency medical services system.
The first step in the process identified the profile for all ambulance trauma responses for the 2002 calendar year. Data will be reported on the state's population ( 4.8 million), total trauma responses $(53,039)$, and number of patients in the following categories: (1) physiological distress $(1,566,3 \%) ;(2)$ significant pattern of injury (11,086, $20.9 \%)$; and (3) mechanism of injury only $(6,664,12.6 \%)$. It is believed that the size and completeness of this data set makes it unique.

The second step was to further analyze the mechanism of injury only category: first, identify the frequency of patients assigned into each of the traditional trauma mechanism categories, and then calculate their predictive values. This analysis identified only two criteria, which demonstrated statistical significance, MCA $>60 \mathrm{~km} / \mathrm{h}$, and a fall from a height $>5 \mathrm{~m}$; however, these criteria are of doubtful clinical or operational significance.

This paper will provide further analysis of this category and discuss the implications and limitations of the study. It is believed that this is the largest ambulance-based analysis of the predictive value of mechanism of injury in prehospital trauma triage and the outcomes will be of international significance in contributing to the evidence-base of prehospital trauma triage.

Keywords: criteria; database; emergency medical services (EMS); falls; mechanism of injury; prediction; prehospital; severity; trauma; triage

Prebosp Disast Med 2005;20(3):s147

\section{Poster Presentations - Tsunami}

\section{Experiences in Sumatra following the Tsunami in Indonesia and the Okushiri Tsunami in Japan Yasufumi Asai, MD; Norifumi Ninomiya, MD; Lee Kwoni, MDC; Kazumi Yano, MD; Tatsuro Kai, MD; Yasubiro Yamamoto, $M D$ \\ Department of Traumatology and Critical Care Medicine, Sapporo Medical University, Japan International Cooperation Agency (JICA), Japan}

Introduction: Following a request by the Government of Indonesia, the Government of Japan sent the Japan Disaster Relief (JDR) Medical Team to Sumatra. Also, there was a big tsunami in Okushiri Island in Japan in 1993.

Objective: The JDR provided medical assistance in a field hospital set up in Banda Aceh from 02-19 January 2005. The experiences obtained from this tsunami were compared with those from the Okushiri tsunami.

Results and Discussion:

1. In Banda Aceh, $>100,000$ residents died by drowning. The first team, which consisted of 22 members including four doctors, treated 1,436 patients during the 10 days. One-quarter of these patients were victims of trauma with infected wounds. Others had respiratory complaints associated with drinking seawater, skin diseases related to bad sanitation, or acute stress disorders.

2. On 12 July 1993, an earthquake and subsequent tsunami struck Okushiri Island in north Japan. A total 